
8 Research Square
Preprints are preliminary reports that have not undergone peer review.
They should not be considered conclusive, used to inform clinical practice, or referenced by the media as validated information.

\title{
Transforaminal Percutaneous Endoscopic Discectomy in the Treatment of Adolescent Lumbar Disc Herniations With Posterior Ring Apophysis Separation
}

changkun zheng ( $\sim$ zck20212021@163.com )

Fuzhou Second Hospital affiliated to Xiamen Universtiy

Zhong Liao

Fuzhou Second Hospital affiliated to Xiamen University

\section{Research Article}

Keywords: percutaneous endoscopic discectomy, lumbar disc herniation, transforaminal, posterior ring apophysis fracture

Posted Date: September 27th, 2021

DOl: https://doi.org/10.21203/rs.3.rs-873681/v1

License: (c) (i) This work is licensed under a Creative Commons Attribution 4.0 International License.

Read Full License 


\section{Abstract}

Objective: To assess the efficacy of transforaminal percutaneous endoscopic discectomy in the treatment of adolescent lumbar disc herniations with posterior ring apophysis separation

Methods: Overall 23 cases of adolescent lumbar disc herniations with posterior ring apophysis separation were treated with the procedure of transforaminal percutaneous endoscopic discectomy between January 2016 and December 2019. Preoperative and postoperative (6 week, 6 month and 12 month) clinical outcome data (back and leg VAS and Macnab criteria) were collected along with clinical assessments of motor strength (graded 0-5).

Results All patients were discharged to home on the same day of surgery. The average leg Visual Analog Scale improved from $8.7 \pm 1.5$ to $2.0 \pm 0.5$ ( $p<0.005$ ). Fifteen patients had excellent outcomes, six had good outcomes, two had fair outcomes, and no had poor outcomes, according to the Macnab criteria. Ten of eleven patients had excellent or good outcomes, for an overall success rate of $91.3 \%$. No patients required reoperation. There were no incidental durotomies, infections, vascular or visceral injuries. There was 1 complication, a case of leg numbness caused by ganglion injury. The numbness improved after 3 weeks. After 2 months, it was obvious that the total area of numbness in the legs had become smaller. At last follow-up, the patient had no pain, and only a few areas with numbness remained and did not affect the patient's activities of daily living.

Conclusion:Transforaminal percutaneous endoscopic discectomy achieve satisfactory results for adolescent lumbar disc herniations with posterior ring apophysis separation.

\section{Introduction}

In the child and adolescent, low back pain and radicular leg pain are not as common a complaint as in adults. The vertebral ring apophysis ossifies at age between 6 and 13 years and fuses with the vertebral body by skeletal maturation(about 18 years old).[1]Before the vertebral ring apophysis fusion, a weak point exists between the ring apophysis and the adjacent vertebral body.[2] If disc herniation occurs at this weak point with resultant isolation and nonfusion of the ring apophysis, an anterior or posterior limbus vertebra is produced. Lumbar posterior ring apophysis fracture occurs when the bony fragment splits from the posterior edge of the superior or inferior endplate of the vertebral body before complete fusion between the ring apophysis and vertebral endplate. Posterior ring apophysis separation is often associated with lumbar disc herniation, resulting in low back pain and limited mobility. [3] Surgical treatment is recommended if conservative treatment has not been effective. To our knowledge, there is no report of transforaminal percutaneous endoscopic discectomy in the treatment of adolescent lumbar disc herniations with posterior ring apophysis separation. In this report, we describe the clinical course and transforaminal percutaneous endoscopic discectomy in the treatment of adolescent lumbar disc herniations with posterior ring apophysis separation. 


\section{Materials And Methods}

After the Institutional Review Board approval, from January 2016 and December 2019, 23 consecutive adolescent lumbar disc herniations with posterior ring apophysis separation were enrolled in the study. There were fifteen males and eight females. The mean age at the time of diagnosis and treatment initiation was 14.6 years (range, 13-18y). All patients were treated with nonsteroidal anti-inflammatory medications, steroid combined with bedrest, and there was no improvement in symptoms after treatment for 3 months. After undergoing dynamic lumbar radiography, thin-section computed tomography, and magnetic resonance imaging examinations, all patients were diagnosed with simple lumbar disc herniations with posterior ring apophysis separation, including 2 in segments L3-L4, 15 in segments L4L5, and 6 in segments L5-S1 (Table 1).

Preoperative

All patients underwent transforaminal percutaneous endoscopic discectomy. All patients underwent preoperative MRI and CT of the lumbar spine. Plain X-rays (standing antero-posterior, lateral views) were obtained preoperatively and 1 year postoperatively. Preoperative and postoperative ( 6 week, 6 month and 12 month) clinical outcome data (back and leg VAS and Macnab criteria) were collected along with clinical assessments of motor strength (graded $0-5$ ), light touch, pain, reflexes, and proprioception. All of the cases were performed by the same surgeon at a single institution. Local anesthesia was used. Patients were informed that they may be felt discomfort and pain during surgery. A radiolucent operating bed and C-arm were used. A solution of $3000 \mathrm{~mL}$ normal saline plus $0.5 \mathrm{mg}$ epinephrine was prepared for intraoperative continuous infusion.

\section{Operative}

The patient was placed on the operating bed in the lateral position. The design of the accurate route from the skin to the herniated disk and appropriate patient position are key for surgical outcomes. The patient was given selective local anesthesia using lidocaine with different concentrations for various layers. Aspiration localization was determined based on pathological level; for example, when performing an operation at the L4-5 level, the entering point should be 11 to $13 \mathrm{~cm}$ from the posterior midline at the L4 spinous process level. Under direct fluoroscopic visualization, a \#16 spinal needle is used to infiltrate the local anesthetic to the level of the facet joint, such that the spinal needle is left in place as a guide. The correct position of the needle tip is confirmed using both anteriorposterrior and lateral projections. The needle is parallel to the disc space, midway between the endplates, proximal to annulus, with the tip lateral to the medial border of the pedicles. A 3- to 5-mm stab incision is made at the entry site of the needle. A hemostat is used to dilate a tract through the lumbodorsal fascia. The flexible trocar is then placed through the tract adjacent and parallel to the spinal needle (double-needle technique). The correct position of the trocar is confirmed using fluoroscopy, and the spinal needle is withdrawn. Under continuous fluoroscopic visualization, the flexible trocar is advanced to the posterior central aspect of the disc. The outer cannula with dilator is then advanced down the trocar to the annular wall. The dilator is removed and the irrigation/aspiration cannula is advanced 1 to $2 \mathrm{~mm}$ against the annular wall as the 3.0- 
$\mathrm{mm}$ trephine is introduced and advanced to create the annulotomy. A working tube $7.5 \mathrm{~mm}$ in diameter was inserted into the target disk tissue, and an endoscope was placed in the working tube. Various tissues were identified under continuous irrigation, and partial decompression was performed. The degenerated nucleus pulposus was found, and the exiting nerve root was protected. The nerve root was fended off with the working tube. The nerve root was explored and released. Finally, ablation decompression and annuloplasty were performed using bipolar radiofrequency.(figure1,2,3)

\section{Results}

Fifteen male and eight female patients, with an average age of 14.6 years (range, 13-18y), were determined to have single-level lumbar disc herniation with posterior ring apophysis separation. All children received local sedation for surgical anesthesia. Median operative time was 127 minutes (range, 96-260 minutes). Median volume of intraoperative blood loss was $46 \mathrm{~mL}$ (range, 10-90 mL). All patients were discharged to home on the same day of surgery and all patients were followed for a minimum of 12 months. The average leg Visual Analog Scale improved from $8.7 \pm 1.5$ to $2.0 \pm 0.5(p<0.005)$. Five patients had excellent outcomes, five had good outcomes, one had fair outcomes, and no had poor outcomes, according to the Macnab criteria (Table 2). Twenty-one patients had excellent or good outcomes, for an overall success rate of $91.3 \%$. No patients required reoperation. There were no incidental durotomies, infections, vascular or visceral injuries. There was 1 complication, a case of leg numbness caused by ganglion injury. The numbness improved after 3 weeks. After 2 months, it was obvious that the total area of numbness in the legs had become smaller. At last follow-up, the patient had no pain, and only a few areas with numbness remained and did not affect the patient's activities of daily living.

\section{Discussion}

Trauma was identifified as the most common cause, with almost $50 \%$ of patients having a history of trauma. [4] This injury is difficult to diagnosis using plain radiographs or magnetic resonance imaging (MRI). However, this condition can be well recognized using computed tomography (CT) and classified into 4 types: type I, avulsion of the whole posterior limbus; type II, separation fracture of a part of the vertebral body, including the limbus; type III, lateralized chip fractures; and type IV, fractures of the vertebral limbus that span the entire vertebral body. [5 6]Decompression by laminotomy or laminectomy without fusion was the standard treatment of lumbar disc herniations with posterior ring apophysis separation until recently. Regarding the centrally located lesions (types I, II, and IV) causing canal or foraminal stenosis, extensive resection of the superior or inferior adjacent lamina was ecessary. Therefore, bilateral laminotomy or full laminectomy has been suggested by many investigators to be required to remove the large and extensive fragment. Regarding type III, the unilateral surgical procedure could achieve suffificient decompression and function improvement for the lateralized chip fractures, including semi-laminotomy, laminectomy, and microendoscopic discectomy. Nevertheless, the posterior spinal structure destruction resulting from open surgery could affect the postoperative results such as 
residual low back pain, iatrogenic instability, and epidural scarring. Hence, it is essential to achieve suffificient neural decompression and minimize the surgical trauma, especially for adolescents.

The initial management of lumbar disc herniation in adolescents is the same as in adults and consists of conservative treatment comprising bed rest, analgesic and anti-inflammatory agents, physical therapy and limitation of physical activities $[8,9]$. Conservative measures are considered before surgery unless lumbar disc herniation affects the patient's motor and neurological functions or causing a truly incapacitating pain[9]. There were also reports of successful results from the use of epidural steroid injections as a part of conservative treatments for pediatric lumbar disc herniation [10]. Adolescents are less responsive to conservative treatment than adults and this is mainly attributed to the viscosity and high elasticity of the inter-vertebral disc in adolescents compared with that in adults. Very few clinical studies have reported successful conservative treatment of lumbar disc herniation in adolescents. Kurth et al. [11] compared outcome of conservative treatment with surgical treatment for 33 children (18 conservatively and 15 surgically treated cases) with a follow-up of 5.4 years, and found no significance between the two groups. DeLuca et al. [9], however, found that surgical treatment lead to a significant better outcome than conservative treatment by carrying out a similar study on 31 children (8 conservative, 23 surgical) with a 6-year follow-up. Regardless of the controversy, it has been widely agreed by most authors that conservative treatment is not as effective for children lumbar disc herniation as it is for adults $[7,9]$.

Operative treatment of lumbar disc herniation should be considered when incapacitating pain disables the patient or neurological deficit or after failure of conservative treatment. However, most adolescent lumbar disc herniations with posterior ring apophysis separation do not respond well to conservative treatment and thus require operative intervention. Adolescents who had neurological deficits and progressive nerve root compression underwent early surgical intervention resulting in excellent recovery outcome.[11] Removal of lumbar disc herniation in adolescents was usually difficult because of its viscous and slippery consistency [12]. However, results of surgical treatment in $98 \%$ of children are good or excellent, which is much better than that reported in other age groups $[11,12]$. The surgical approach should be individualized and the amount of bone removal should be balanced against the risk of developing subsequent spinal deformity in the growing child.

Various techniques have been proposed for the operative treatment of lumbar disc herniations with posterior ring apophysis separation. These include midline incisions and subperiosteal muscle dissections with hemi-laminectomy and partial or complete facetectomy or paramedian incisions with intermuscular dissections for an intertransverse approach; and combined approaches for lumbar disc herniation Adhesion, scar compression, and dura mater fibrosis often occur postoperatively, which may lead to related symptoms. Instability and severe back pain have been reported after an interlaminar approach. These sequestrated fragments may be missed even after full facetectomy and therefore are the cause of persistent radicular pain [13]. Together, minimal internal tissue damage and a smaller surgical incision (approximately $7 \mathrm{~mm}$ ) reduces the revalidation period, minimizes scar tissue, and 
reduces complications. Intraoperative bleeding is reduced, and hemostasis can be expected postoperatively. It encourages fast wound healing and reduces the risk of infection[14].

The clinical symptoms of lumbar disc herniations with posterior ring apophysis separation were mainly caused by the herniated disc as shown by MRI. Because the texture of the bony fragment was hard with sharp edges, it was proper to resect the herniated disc first to obtain a wider operative space, providing the advantages of avoiding nerve root injury and dural tears. After discectomy, burrs and a rongeur should be used to excise the bony fragment step-by-step under visual control. All the loose and mobile fragments should be removed. In contrast, whether the fixed and immobile fragments should be removed will depend on whether they had compressed the nerve root. Those fragments resulting in compression should be removed completely. However, if the fragments are not causing compression, they can be retained to prevent extensive damage to the intervertebral structure, as suggested in some previous studies.

\section{Conclusion}

The clinical symptoms of lumbar disc herniations with posterior ring apophysis separation were mainly caused by the herniated disc. Transforaminal percutaneous endoscopic discectomy may be indicated for adolescent lumbar disc herniations with posterior ring apophysis separation.

\section{Declarations}

Funding: No

Conflict of Interest:

The authors declare that they have no conflict of interest

\section{References}

1. Benzakour T, Igoumenou V, Mavrogenis AF et al (2019) Current concepts for lumbar disc herniation. Int Orthop 43(4):841-851

2. Akhaddar A, Belfquih $\mathrm{H}$, Oukabli M et al (2011) Posterior ring apophysis separation combined with lumbar disc herniation in adults: a 10-year experience in the surgical management of 87 cases. $\mathrm{J}$ Neurosurg Spine 14:475-483

3. Wang X, Zeng J, Nie H, Chen G, Li Z, Jiang H, Kong Q, Song Y, Liu H (2014 May) Percutaneous endoscopic interlaminar discectomy for pediatric lumbar disc herniation. Childs Nerv Syst 30(5):897-902

4. Martínezlage JF, Poza M, Arcas $P$ (1998) Avulsed lumbar vertebral rim plate in an adolescent: trauma or malformation? Childs Nerv Syst 14:131-134 
5. Epstein NE, Epstein JA, Mauri T (1989) Treatment of fractures of the vertebral limbus and spinal stenosis in five adolescents and five adults. Neurosurgery 24:595-604

6. Takata K, Inoue S, Takahashi K et al (1998) Fracture of the posterior margin of a lumbar vertebral body. J Bone Joint Surg Am 70:589-594

7. Slotkin Jonathan R, Mislow John MK, Day Arthur L et al (2007) Pediatric disk disease. Neurosurg Clin N Am 18(4):659-667

8. Chen Y, Song R, Huang W, Chang Z (2018 Nov) Percutaneous endoscopic discectomy in adolescent lumbar disc herniation: a 3- to 5-year study. J Neurosurg Pediatr 23(2)(2):251-258

9. Benzakour A, Benzakour T (2019) Lumbar disc herniation: long-term outcomes after mini-open discectomy. Int Orthop 43(4):869-874

10. Kumar Raj, Kumar V, Das NK et al (2007) Adolescent lumbar disc disease: findings and outcome. Childs Nerv Syst 23(11):1295-1299

11. Tsutsumi S, Yasumoto Y, Ito M (2011 Jul) Idiopathic intervertebral disk calcification in childhood: a case report and review of literature. Childs Nerv Syst 27(7):1045-1051

12. Huynh TR, Lagman C, Sweiss F, Shweikeh F, Nuño M, Drazin D (2017 Sep) Pediatric spondylolysis/spinal stenosis and disc herniation: national trends in decompression and discectomy surgery evaluated through the Kids' Inpatient Database. Childs Nerv Syst 33(9):1563-1570

13. Voyadzis J-M, Gala VC, Sandhu FA et al (2010) Minimally invasive approach for far lateral disc herniations: results from 20 patients. Minim Invasive Neurosurg 53(3):122-126

14. Zheng C, Wu F, Cai L (2016) Transforaminal percutaneous endoscopic discectomy in the treatment of far-lateral lumbar disc herniations in children. Int Orthop 40(6):1099-1102

\section{Tables}

TABLE 1 Characteristics of 23 adolescent lumbar disc herniations with posterior ring apophysis separation

\begin{tabular}{lcc} 
Characteristic & No. of Patients (\%) \\
\hline male/female & $1: 0.53(15 / 8)$ \\
\hline level & & \\
\hline L3-4 & & $2(8.7)$ \\
\hline L4-5 & 15 & $(65.2)$ \\
\hline L5-S1 & 6 & $(26.1)$
\end{tabular}

TABLE 2 Outcome after endoscopic discectomy 
Outcome

No. of Patients (\%)

\begin{tabular}{lll}
\hline Macnab criteria & & \\
\hline excellent & 15 & $(65.2)$ \\
\hline good & 6 & $(26.1)$ \\
\hline fair & & $2(8.7)$ \\
\hline poor & 0 & $(0)$
\end{tabular}

Figures

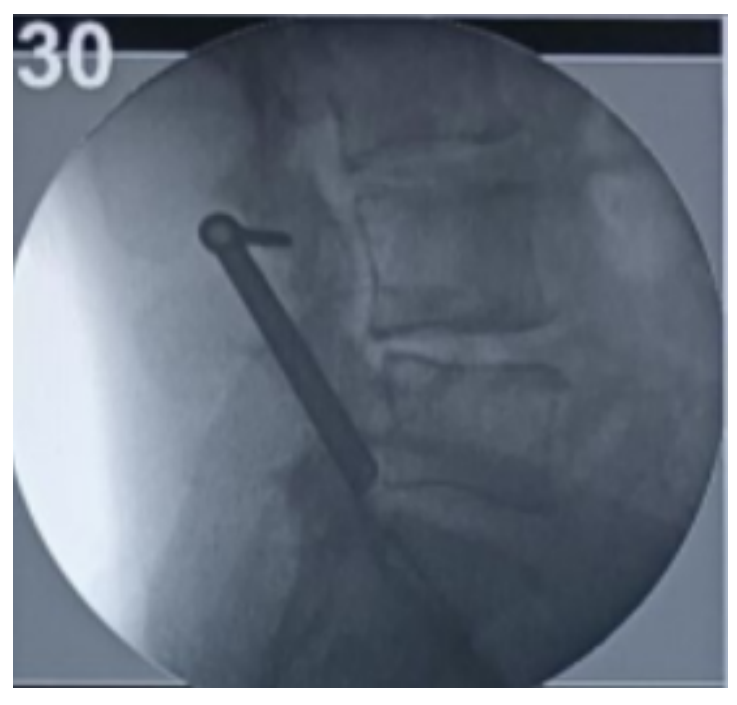

Figure 1

Lateral radiograph showing placement of the working tube.

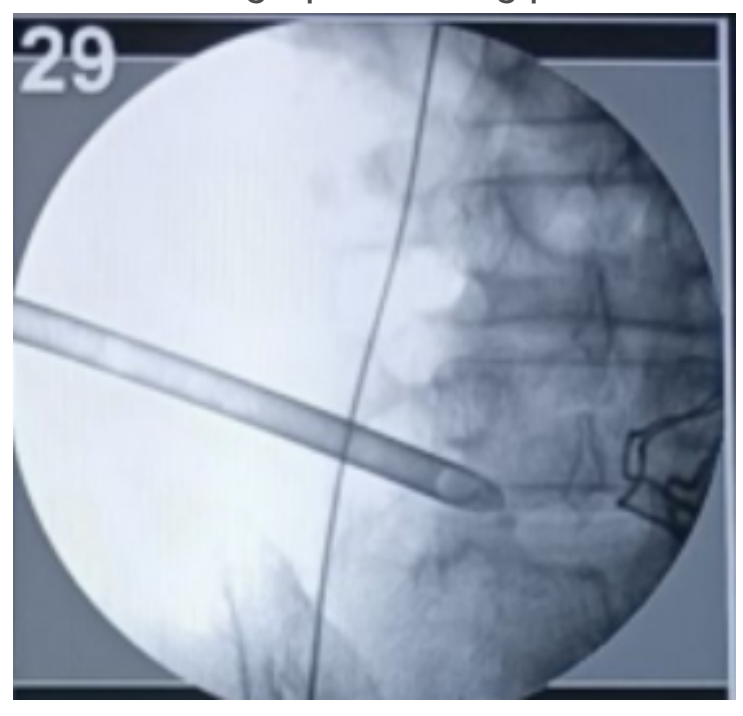

Figure 2 


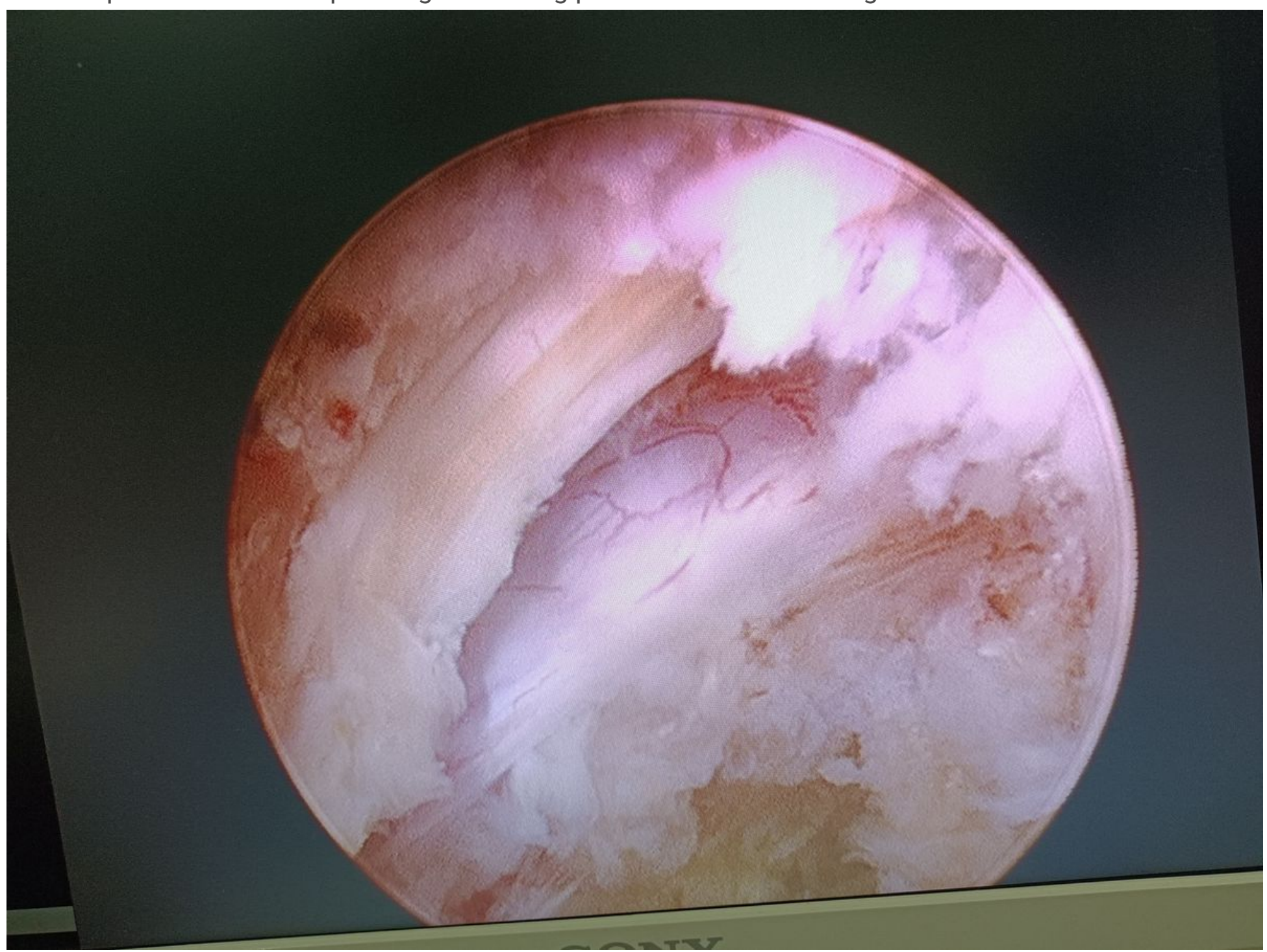

\section{Figure 3}

An intraoperative endoscopic view of the final phase of procedure during percutaneous endoscopic discectomy . The lateral margin of nerve root is well exposed after full decompression. 